\title{
Clinical usefulness of the 16-slice computed tomography coronary angiography for evaluation of early phase intracoronary stent patency
}

\author{
Serkan Yüksel ${ }^{a *}$, Mahmut Şahin ${ }^{a}$, Muzaffer Elmalı ${ }^{\mathrm{b}}$, Ayşegül İdil Soylu ${ }^{\mathrm{a}}$, Çetin Çelenk ${ }^{\mathrm{b}}$, Sabri Demircan ${ }^{\mathrm{a}}$, Okan Gülel ${ }^{\mathrm{a}}$, \\ Erdoğan Yaşar ${ }^{\mathrm{a}}$, Özcan Yilmaz
}

${ }^{a}$ Department of Cardiology, Faculty of Medicine, Ondokuz Mayis University, Samsun, Turkey

${ }^{b}$ Department of Radiology, Faculty of Medicine, Ondokuz Mayis University, Samsun, Turkey

\begin{tabular}{|c|c|}
\hline ARTICLE INFO & ABSTRACT \\
\hline $\begin{array}{ll}\text { Article History } \\
\text { Received } & 02 / 08 / 2012 \\
\text { Accepted } & 30 / 08 / 2012\end{array}$ & $\begin{array}{l}\text { Coronary artery stenting is currently treatment of choice for management of coronary } \\
\text { artery disease. Stent restenosis is the most important problem during follow up. } \\
\text { Conventional coronary angiography is the gold standart for assessment of intracoronary } \\
\text { stent patency. It is an invasive method and even though rare, has some significant risks. }\end{array}$ \\
\hline $\begin{array}{l}\text { * Correspondence to: } \\
\text { Serkan Yüksel } \\
\text { Department of Cardiology, } \\
\text { Faculty of Medicine, } \\
\text { Ondokuz Mayis University, } \\
\text { Samsun, Turkey } \\
\text { e-mail: serkan.yuksel@omu.edu.tr }\end{array}$ & $\begin{array}{l}\text { For this reason, noninvasive imaging methods are necessary to evaluate stent patency. } \\
\text { Noninvasive methods such as the exercise test, myocardial perfusion scintigraphy and } \\
\text { stress echocardiography could not reach enough diagnostic accuracy. Multislice computed } \\
\text { tomography (MSCT) has been under investigation for stent restenosis detection. Aim of } \\
\text { this study is to investigate usefulness of the } 16 \text {-slice CT for evaluation of stent patency in } \\
\text { patients with suspicion of stent restenosis. Thirty six patients were included in the study } \\
\text { and } 16 \text {-slice CT and conventional coronary angiographies were performed in all patients. } \\
\text { The results of } 16 \text {-slice CT and conventional coronary angiography were compared. } \\
\text { Sufficient or good quality imaging with 16-slice CT angiography was obtained in } 69 \%\end{array}$ \\
\hline $\begin{array}{l}\text { Keywords: } \\
\text { Conventional coronary angiography } \\
\text { Coronary artery disease } \\
\text { Intracoronary stent } \\
\text { Multislice computed tomography } \\
\text { Stent patency } \\
\text { Stent restenosis }\end{array}$ & $\begin{array}{l}\text { correct localization for all of the detected stents. Stent lumen could be assessed in } 30 \\
\text { (61\%) stents and according to the results of luminal assessment, sensitivity, specificity, } \\
\text { positive predictive value (PPV), negative predictive value (NPV) and diagnostic accuracy } \\
\text { of } 16 \text {-slice MSCT were calculated as } 33 \%, 95 \%, 75 \%, 77 \% \text { and } 77 \% \text {, respectively. } \\
\text { According to these results, the diagnostic performance of } 16 \text {-slice CT angiography for } \\
\text { detection of stent restenosis was relatively low. However, the assessment of relatively } \\
\text { small number of stents because of insufficient heart rate control did not allow reliable } \\
\text { and precise evaluation. Our results showed that diagnostic capacity of } 16 \text {-slice CT } \\
\text { angiography for detection of coronary stent restenosis is limited. }\end{array}$ \\
\hline
\end{tabular}

\section{Introduction}

Intracoronary stent implantation is being used as a primary treatment method in coronary artery disease with increasing frequency. Intracoronary stent restenosis or occlusion is an expected finding in the first six months after intracoronary stenting in patients with recurrent ischemic symptoms (Antoniucci et al., 1998). Therefore; in order to guide treatment in this patient group; coronary artery anatomy, ventricular function and the severity of restenosis are need to be evaluated.

Conventional coronary angiography is the traditional gold standard method for the imaging of the coronary arteries. There are complications of conventional coronary angiography such as; the arterial access site trauma, pseudoaneurysm formation, cardiac arrhythmia, stroke, myocardial infarction, coronary artery dissection, embolism, or renal failure due to contrast media (Scanlon et al., 1999). These morbidities associated with coronary angiography, invasive aspect of procedure and need for hospitalization restricts the application of this method in patients with possible stent restenosis.

Evaluation of coronary anatomy with multislice computed tomography (MSCT) angiography is an emerging imaging modality. With the increase of the detector number, coronary artery imaging with enhanced temporal and spatial resolution can be obtained by MSCT.

In this study, we aimed to evaluate the usefulness of 16-slice computed tomography (CT) in comparison to con- 
ventional coronary angiography which is a gold standart in determination of stent restenosis in patients with suspicion of early stent restenosis. In this study, the restenosis or patency of intracoronary stents implanted within last six months were evaluated with 16-slice CT angiography and conventional coronary angiography.

\section{Materials and methods \\ Patient selection}

Thirty-six patients (30 male; 6 females, mean ages $56 \pm 11$ years) in whom intracoronary stents implanted within last six months were admitted to Ondokuz Mayis University Hospital and Cardiology Clinic with the diagnosis of possible stent restenosis were included in this study.

Patients with arrhythmias, especially atrial fibrillation, impaired renal functions (creatinine level $>2 \mathrm{mg} / \mathrm{dL}$ ), allergic to iodinated contrast media, pregnancy, severe chronic obstructive pulmonary disease, severe heart failure (New York Heart Association Class III-IV), acute coronary syndromes and claustrophobia were excluded from the study. A written informed consent form was signed by each patient included in the study. The research protocol was approved by the Ethics Committee of the Ondokuz Mayis University, Faculty of Medicine.

\section{Conventional coronary angiography}

Selective coronary angiographies were performed after right femoral artery puncture by using the Seldinger technique. The right and left coronary arteries were imaged using oblique positions in the cranial and caudal angulations. Coronary angiographies were performed by Siemens AXIOM Artis (Siemens Medical Diagnostics, Siemens AG, Germany) device. Immediately after the procedure, the results of coronary angiographies were evaluated by the operators. Stent patency and restenosis were evaluated by two criteria.

1. Patent: Absence of restenosis or noncritic stenosis $(<50 \%)$.

2. Stent restenosis: More than $50 \%$ stenosis in stent or total occlusion without distal flow.

The Sixteen-Slice computed tomography coronary angiography protocol and image processing

Patients underwent 16-slice CT angiography in Computed Tomography Unit of Ondokuz Mayis University Hospital. Imaging was performed by 16-slice CT scanner (Aquilion 16 system, Toshiba Medical System Corporation, Japan) and accompanied by ECG monitorization. Sixteen-slice CT detector collimation was $16 \times 1 \mathrm{~mm}, \mathrm{kVp} 135$, mAs 350 , pitch 0.25 , rotation time 0.4 , slice thickness $1 \mathrm{~mm}$, and reconstruction interval $0.5 \mathrm{~mm}$, respectively. An intravenous line from antecubital vein was inserted in all patients, and $120 \mathrm{ml}$ nonionic iodinated contrast material was given intravenously, via an automatic injector (Ulrich Medizintechnik, Missouri) with a speed of $4 \mathrm{ml} / \mathrm{sec}$.

Reconstructed images in different phases of the R-R interval by using the retrospective electrocardiography (ECG) gating were obtained from the axial raw images. From these images, at least two best phases were selected and axial, maximum intesity projection (MIP), multiplane reformatted (MPR) and 3-dimensional images obtained from the last images were evaluated in Vitrae Workstation.
Sixteen-slice CT coronary angiography images were evaluated separately by two independent observers. Observers stated the assessibility of stent lumen and location of the stent in the 16-slice CT images. Assessible stent is defined as having a visible lumen, evaluable contrast density in lumen without partial volume effect, metal artifact and calcification. Observers also reported the reasons of inadequate stent imaging. Two criterias were used in evaluation of stent restenosis in 16-slice CT (Kitagawa et al, 2006).

1. Patent: Homogenous and visual similarity of contrast media density of stent lumen to the reference vessel (proximal and distal to the stented area).

2. Stent restenosis: Invisibility of distal flow in stented area of coronary artery (occluded), or a low density in the stent lumen compared to reference vessel.

\section{Statistical analysis}

Statistical analysis was performed by taking the results of conventional invasive coronary angiography as reference. In order to determine the diagnostic efficacy of 16-slice CT coronary angiography; the sensitivity, specificity, PPV, NPV, and diagnostic accuracy were calculated. In addition, the aggreement between two observers was evaluated by the Kappa test. $\mathrm{p}$ value $<0.05$ was considered as statistically significant.

\section{Results}

\section{Clinical characteristics of the patients}

Thirty-six patients (30 males, 6 females, mean age $56 \pm 11$ years) scheduled for coronary angiography with the diagnosis of stent restenosis were included in the study. The mean duration between the intracoronary stent implantation and conventional coronary angiography was $127 \pm 79$ days, the median duration between 16-slice computed tomographic angiography and conventional coronary angiography was 5 days (ranges from 1 to 21 days), respectively. Clinical characteristics of the patients are presented in Table 1 .

Table 1. Clinical characteristics of the patients

\begin{tabular}{lc} 
Clinical characteristics & Number $\mathbf{( \% )}$ \\
\hline Age (year) & $56 \pm 11$ \\
Gender & \\
$\quad$ Male & $30(83 \%)$ \\
$\quad$ Female & $6(17 \%)$ \\
HT history & $14(39 \%)$ \\
DM history & $5(14 \%)$ \\
Smoking history & $25(69 \%)$ \\
Hyperlipidemia & $10(28 \%)$ \\
Familial history of CAD & $17(47 \%)$ \\
BMI (kg/m $\left.{ }^{2}\right)$ & $26.9 \pm 4.9$ \\
Creatinine (mg/dL) & $0.9 \pm 0.3$ \\
EF (Simpson) (\%) & $50.5 \pm 13.4$ \\
\hline HT: Hypertension; DM: Diabetes mellitus; CAD: Coronary artery \\
disease; BMI: Body mass index; EF: Ejection fraction.
\end{tabular}

There were 49 intracoronary stents in 36 patients. The median diameter of intracoronary stents was $3.00 \mathrm{~mm}(2.50$ $\mathrm{mm}$ to $4.00 \mathrm{~mm})$, and the median stent length was $15 \mathrm{~mm}$ (9 $\mathrm{mm}$ and $32 \mathrm{~mm})$. Thirty-five (72\%) stents were implanted to left anterior descending (LAD) artery, 5 (10\%) stents to circumflex $(\mathrm{Cx})$ artery, and $9(18 \%)$ stents to right coronary artery (RCA). 
Table 2. The distribution of intracoronary stents according to implanted coronary arteries, diameter and stent material

\begin{tabular}{lcccc} 
Stent characteristics & LAD & CX & RCA & Total \\
\hline Stent diameter $(\mathbf{m m})$ & 5 & 1 & 1 & $7(14 \%)$ \\
2.50 & 6 & 0 & 1 & $7(14 \%)$ \\
2.75 & 15 & 1 & 5 & $21(43 \%)$ \\
3.00 & 6 & 2 & 1 & $9(19 \%)$ \\
3.50 & 3 & 1 & 1 & $5(10 \%)$ \\
4.00 & & & & \\
Stent material & 18 & 3 & 8 & $29(59 \%)$ \\
316 L stainless steel & 10 & 1 & 0 & $11(23 \%)$ \\
Dylin coated 316 L stainless steel Silicon & 3 & 1 & 1 & $5(10 \%)$ \\
Coated 316L stainless steel & 1 & 0 & 0 & $1(2 \%)$ \\
Cobalt cromium & 3 & 0 & 0 & $3(6 \%)$ \\
Unknown & & & & \\
\hline
\end{tabular}

LAD: Left anterior descending, Cx: Circumflex, RCA: Right coronary artery

There were 12 different stent brands and 4 different stent materials. The distribution of intracoronary stents according to implanted coronary arteries, diameter and material was presented in Table 2 .

\section{Assessment of intracoronary stents by conventional coro- nary angiography}

Conventional coronary angiographies were performed by the responsible operators and no complication was observed during and after the procedures. Thirty seven (76\%) stents were patent, and stent restenosis were determined in 12 (24\%) stents. Stent restenosis was detected in 6 , whereas remaining 6 stents were totally occluded. When the stents were assessed according to implanted vessel; 23 (70\%) of 33 stent were patent in LAD artery, 5 (87\%) of 6 were patent in Cx artery, 9 $(90 \%)$ of 10 were patent in RCA. Stent restenosis was detected in $36 \%$ of stents with a diameter of less than $3.00 \mathrm{~mm}$, and $20 \%$ of the stents with a diameter of $3.00 \mathrm{~mm}$ and more.

\section{Assessment of intracoronary stents by the 16-slice com- puted tomography angiography}

The 16-slice CT coronary angiographies were performed without any complication in 36 patients included in the study. The mean heart rate of patients at the beginning of the 16-slice CT coronary angiography scans was $73 \pm 9$ beats / min. Eighty nine percent $(32 / 36)$ of the patients were already on oral beta-blocker treatment before the 16-slice CT coronary angiography.

The 16-slice CT coronary angiography imaging quality was inadequate in $11(30.6 \%)$ patients (Fig. 1), adequate in 21 (58.3\%) patients, and, high-quality in $4(11.1 \%)$ patients (Fig. $2)$. The causes of inadequate imaging were reported as dense calcification of the coronary arteries in $55 \%$ of patients, motion artifact due to insufficient heart rate reduction in $45 \%$ of the patients.

\footnotetext{
Assessment of intracoronary stent lumens by the 16-slice computed tomography angiography

The lumens of 30 (61\%) intracoronary stent were appropriate for assessment. In 19 (39\%) stents, the lumens were invisible. According to the coronary arteries; 13 (37\%) of 35 stents in LAD, $3(60 \%)$ of 5 stents in Cx, and $3(33 \%)$ of 9 stents in RCA could not be assessed.
}

Assessment of intracoronary stent patency by 16-slice computed tomography angiography

Twenty six (87\%) intracoronary stents were assessed as patent and four $(13 \%)$ stents were assessed as restenotic by the 16-slice CT coronary angiography. There were 19 patents $(86 \%)$ and $3(14 \%)$ restenotic stents in the LAD artery. Two $(100 \%)$ and five $(83 \%)$ stents were patent in the Cx and RCA, respectively. There was $1(17 \%)$ restenotic in RCA. With respect to the diameter of the stents, all the 7 stents with visible lumen and having a diameter of less then $3.00 \mathrm{~mm}$ were patent. The assessment of stents with visible lumen and having a diameter of $3.00 \mathrm{~mm}$ and above showed that, 19 (83\%) stents were patent, and $4(17 \%)$ were restenotic.

The comparison of 16-slice computed tomography and conventional coronary angiography results

The 16-slice CT coronary angiography results were assessed by two independent observers after patients recruitment completed and these results were compared with the results of conventional coronary angiography taken as a reference.

First observer evaluated the patency of 30 of 49 intracoronary stent. Twenty six stent $(87 \%)$ were patent, and 4 stent $(13 \%)$ were restenotic. The first observer determined 20 of 21 patent stent and 3 of 9 restenotic stent correctly according to conventional coronary angiography results. Restenosis was detected by conventional coronary angiography in 6 intracoronary stent which were assessed as patent by 16 -slice CT coronary angiography. Additionally, one intracoronary stent was assessed as restenotic by 16-slice CT coronary angiography but, it was patent according to conventional coronary angiography. According to these results, the diagnostic accuracy of the first observer was calculated as $77 \%$.

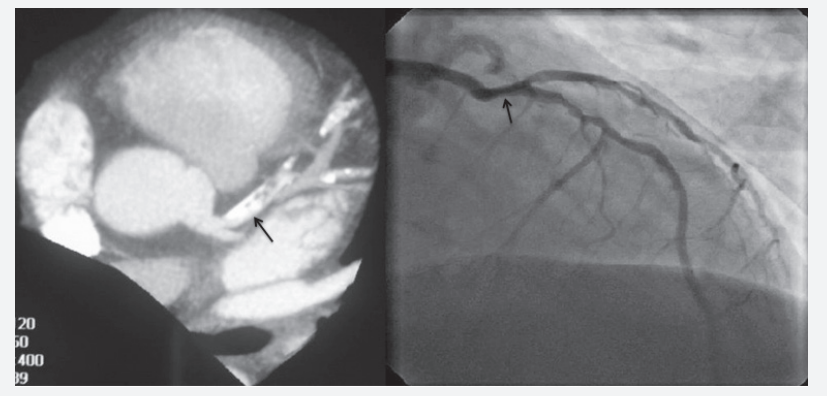

Fig. 1. The 16-slice CT angiographic MIP image of a stent that could not be assessed because of heavy calcification and the conventional coronary angiographic image of same patient.

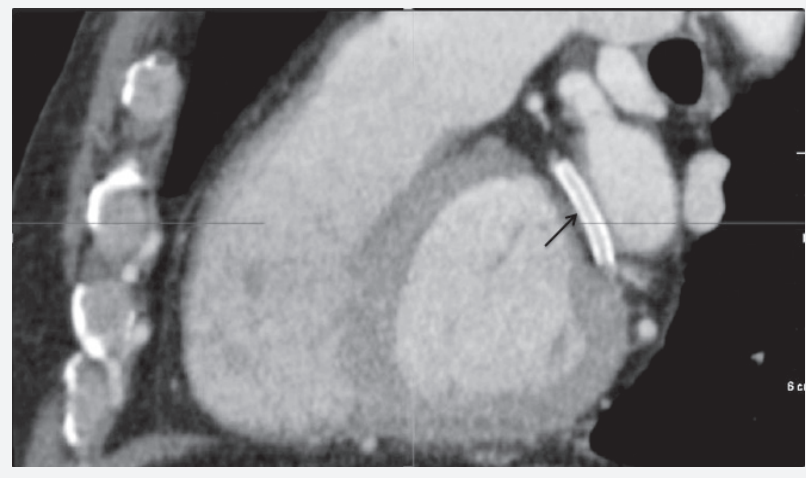

Fig. 2. High quality MPR image of a stent in LAD artery obtained by 16-slice CT angiography. 
Second observer evaluated the patency of $21(43 \%)$ of 49 intracoronary stent. Nineteen stent (91\%) were patent, and 2 stent $(9 \%)$ were restenotic. The second observer determined 15 of 16 patent stent and 1 of 5 restenotic stent correctly according to conventional coronary angiography results.

Restenosis was detected by conventional coronary angiography in 4 intracoronary stent which were assessed as patent by 16-slice CT coronary angiography. Additionally, one intracoronary stent was assessed as restenotic by 16 -slice CT coronary angiography but, it was patent according to conventional coronary angiography. According to these results, the diagnostic accuracy of the second observer was calculated as $76 \%$.

According to the results of the first observer; the sensitivity, specificity, PPV and NPV of 16-slice CT angiography were $33 \%, 95 \%, 75 \%$ and $77 \%$, respectively. According to the results of the second observer, the sensitivity, specificity, positive predictive value (PPV) and negative predictive value (NPV) of 16-slice CT angiography were 20\%, 94\%, 50\% and $79 \%$, respectively. The aggreement between two observers was evaluated by the Kappa test. There was no difference between two observers $(\mathrm{p}=1.00)$. The aggreement between two observers was good (Kappa $=0.638)$ and this result is statistically significant $(\mathrm{p}<0.05)$.

\section{The comparison of 16-slice computed tomography and conventional coronary angiography results according to intracoronary stent diameters}

Intracoronary stents were divided into 2 groups as stents with diameter of less than $3.00 \mathrm{~mm}$ and stents with diameter of $3.00 \mathrm{~mm}$ and higher. The results of both groups obtained by 16-slice CT coronary angiography were compared with the results of conventional coronary angiography. There were 14 stents with diameter less than $3.00 \mathrm{~mm}$. The seven of (50\%) these stents were evaluated by 16 -slice CT coronary angiography and there was no restenosis detected. Restenosis was detected by conventional coronary angiography in 3 of these stents, and the remaining four stents were patent. Therefore, the diagnostic accuracy of 16-slice CT coronary angiography was $57 \%$ in the stents with diameter less than $3.00 \mathrm{~mm}$.

There were 35 stents with diameter of $3.00 \mathrm{~mm}$ and higher and 23 of $(66 \%)$ these stents were evaluated by 16-slice CT coronary angiography. Of these; four (17\%) stents were restenotic, and $19(83 \%)$ stents were patent. In conventional coronary angiography, 3 of the 4 restenotic stents detected by 16-slice CT coronary angiography were found as restenotic and 1 stent was patent. Additionally, conventional coronary angiography detected restenosis in 3 of the 19 patent stents detected by 16-slice CT coronary angiography. Therefore, the diagnostic accuracy of 16-slice CT coronary angiography was $83 \%$ in the stents with diameter $3.00 \mathrm{~mm}$ and higher. According to these results, the sensitivity and PPV of 16-slice CT coronary angiography in stents with diameter less than 3.00 could not be estimated. Specificity and NPV were calculated as $100 \%$ and $57 \%$, respectively. The sensitivity, specificity, PPV, NPV and diagnostic accuracy of 16-slice CT coronary angiography in stents with diameter of $3.00 \mathrm{~mm}$ and higher were $50 \%, 94 \%, 75 \%, 84 \%$ and $83 \%$, respectively.

\section{Discussion}

In this study, the usefulness of 16-slice CT coronary angiog- raphy for the assessment of stent patency in patients with intracoronary stents implanted within last six months was investigated. 16-slice CT coronary angiographies were performed without any complication in all patients. According to data obtained, it can be concluded that 16-slice CT coronary angiography is a safe technique if patient selection is appropriate.

Intracoronary stent implantation is the preferred treatment method in ischemic heart disease and its use is increasing. In a meta-analysis of 11 studies including 5103 patients; the prevalences of angiographic stent restenosis were reported as $5-10 \%$ in DES (drug eluting stents) and 20-35\% in BMS (bare metal stent) (Babapulle et al., 2004). Our study is consistent with other studies as the stent restenosis rate of $24 \%$ was determined.

Altough conventional coronary angiography is accepted as the gold standard for assessment of stent patency, because of the procedure-related risks, and the discomfort, it is not well-accepted method by patients. Functional stress tests are usually applied to patients before conventional coronary angiography. Unfortunately, the accuracy of these tests in the diagnosis of stent restenosis is quite limited. Garzon and Eisenberg (2001) reported poor sensitivity (46\%) and moderate specificity (77\%) of exercise stress test, an acceptable sensitivity (87\%) and specificity (78\%) of myocardial perfusion scintigraphy for the diagnosis of stent restenosis, sensitivity and specificity of stress echocardiography was reported as $63 \%$ and $87 \%$, respectively.

There are studies with non-invasive imaging methods other than 16-slice CT such as electron beam computed tomography (EBCT) and magnetic resonance (MR) angiography to evaluate the intracoronary stent patency. EBCT in recent years has been used as a noninvasive imaging method particularly due to ability to do coronary calcium measurement. However, due to the poor spatial resolution three dimentional imaging of coronary arteries is limited (Möhlenkamp et al., 1999; Pump et al., 2000).

Proximal segments of the coronary arteries can be assessed with MR angiography (Kim et al., 2001). The advantages of MR coronary angiography are lack of radiation exposure and lack of iodinated contrast agent use. However, the limited spatial resolution and a long scan time are the most important limitations. Intracoronary stents especially the stainless-steel stents cause severe artifacts and signal loss and this makes it impossible to display the stent lumen (Hug et al., 2000). Buecker et al. (2002) reported that in some special stent models, MR angiography could show the stent lumen.

The 16-slice CT is superior to other non-invasive imaging methods because of its important features such as; ability to take multiple scans at the same time, very high spatial resolution, relatively short scan time, necessity of shorter breath-hold duration for the patient. With the introduction of 16-slice CT coronary angiography in routine clinical practice, the success of 16-slice CT coronary angiography in determination of significant lesions in coronary arteries was investigated in many studies and it has been reached to wide-range knowledge. However, the number of studies assessing the performance of MSCT angiography to determine intracoronary stent patency is still low. That's why, our study aimed to investigate the usefulness of MSCT coronary angiography in detection of intracoronary stent patency. 
The 16-slice CT coronary angiographic imaging of intracoronary stents is different from imaging of native coronary arteries. Not only the movement of the coronary arteries, but also the metal content of stent affects the detection of in-stent restenosis. The metal content of stents can cause high-intensity artifacts and prevent the imaging of the significant portion of stent lumen. The material and design of the stent affect the amount of artifact. As density increases, artifacts become more pronounced. This effect is less pronounced in the large vessels such as aorta and abdominal branches, but more pronounced and prevents the imaging of lumen in the smaller vessels like coronary arteries (Sun et al., 2009).

Stent lumen visualization with the first-generation 4-slice CT scanners is reported to be impossible (Krüger et al., 2003). Maintz et al. (2003a) reported the thickening of the stent struts by artefacts and therefore a significant reduction in stent lumen diameter in their in-vitro study with 4-slice CT scanners. In addition, the lumen was invisible in Wiktor, WallStent and Nir Royal stents. Krüger et al. (2003) evaluated 32 stents in vivo with 4-slice CT angiography, but they were unable to make any analysis of the stent lumen regardless the type and diameter of stent. Maintz et al. (2003b) reported the visualization of up to $20-40 \%$ of the stent lumen in another study with 4-slice CT angiography. According to these data, it was concluded that the evaluation of intracoronary stent patency with 4-slice CT scanners was not possible in clinical practice.

The 16-slice CT scanners compared to 4-slice CT scanners have more advanced spatial and temporal resolution. The 16-slice CT scanners are more successful than 4-slice CT scanners in evaluation of intracoronary stents with high imaging quality due to thinner slices, high gantry rotation speed and short breath-hold period. The sensitivity and specificity of 16-slice CT scanners in detection of intracoronary stent restenosis ranged between $57-83 \%$ and $88-99 \%$, respectively and the average rate of assessed stents was $86 \%$ (Schuijf et al., 2004; Gilardi et al., 2006; Kitagawa et al., 2006, Watanabe et al., 2006; Kefer et al., 2007; Soon et al., 2007). In our study, the sensitivity of $33 \%$ is quite low compared to other studies, but the specificity is compatible with other studies.

Despite the high spatial resolution due to the advanced technical features, an important limitation of 16-slice CT angiography is the relatively low temporal resolution. This limitation becomes apparent especially in patients with high heart rate and motion artifacts cause deterioration of image quality. Generally, in order to achieve optimal image quality, heart rate is required to less than 70 beat/minutes and for this purpose; eligible patients premedicated with beta-blocker drugs. Motion artifacts are even bigger problem in patients with arrhythmias and contraindications to beta-blockers. The stents implanted to $\mathrm{Cx}$ artery and RCA are more vulnerable to motion artifacts. By the development of new generation CT scanners (particularly 64-slices and more) with higher temporal resolution, this effect is dramatically reduced (Sun et al., 2012).

In our study, 23 patients $(64 \%)$ with heart rates higher than 70 beats/min received the intravenous premedication. However, unlike many other studies, there was no upper heart rate limit established as a criterion for exclusion. In our study, the mean heart rate at the beginning of the scan was $73 \pm 9$ beats / minute. This heart rate is quite high compared to the other studies. Nowadays, more and more widely used 64 and higher slice CT scanners with high gantry rotation speeds and capacity of taking more slice allow to obtain higher quality images in patients with high heart rates.

In a meta-analysis of 15 studies, 1175 intracoronary stent were assessed with the 16 and 64-slice CT scanners, and the average sensitivity and specificity were reported as $84 \%$ and $91 \%$, respectively (Hamon et al., 2008). In our study, 33\% sensitivity of 16-slice CT angiography is below the sensitivities of other studies but $95 \%$ specificity level is comparable to other studies. However, only nine restenotic stents were assessed within 30 stents. The evaluation of 16-slice CT angiography in intracoronary stent restenosis detection with only 9 stents is not reliable enough to draw conclusions. For this reason, the low number of the total and restenotic stents assessed is one of the limitations of our study.

The patients included in this study underwent 16-slice CT angiography and conventional coronary angiography with the clinical indications. This study was not planned in randomized, prospective design. The potential bias caused by results of 16-slice CT and conventional coronary angiography performed with clinical indications are another limitation of the study.

In conclusion; the 16-slice CT angiography seems unlikely to take place of conventional coronary angiography in the evaluation of stent restenosis in clinical practice according to the data obtained from this study. It is difficult to reach a reliable conclusion about performance of 16-slice CT angiography in assessment of stent restenosis because of the low number of stents and low restenosis rate. More comprehensive studies including higher number of stents and high restenosis rate are needed.

\section{REFERENCES}

Antoniucci, D., Valenti, R., Santoro, G.M., Bolognese, L., Trapani, M., Cerisano, G., Boddi, V., Fazzini, P.F., 1998. Restenosis after coronary stenting in current clinical practice. Am. Heart J. 135, 510-518.

Babapulle, M.N., Joseph, L., Bélisle, P., Brophy, J.M., Eisenberg, M.J., 2004. A hierarchical Bayesian meta-analysis of randomised clinical trials of drug-eluting stents. Lancet. 364, 583-591.

Buecker, A., Spuentrup, E., Ruebben, A., Günther, R.W., 2002. Artifact-free in-stent lumen visualization by standard magnetic resonance angiography using a new metallic magnetic resonance imaging stent. Circulation. 105, 1772-1775.

Garzon, P.P., Eisenberg, M.J., 2001. Functional testing for the detection of restenosis after percutaneous transluminal coronary angioplasty: A meta-analysis. Can J. Cardiol. 1, 41-48.

Gilardi, M., Cornily, J.C., Pennec, P.Y., Le Gal, G., Nonent, M., Mansourati, J., Blanc, J.J., Boschat, J., 2006. Assessment of coronary artery stents by 16 slice computed tomography. Heart. 92, 58-61.

Hamon, M., Champ-Rigot, L., Morello, R., Riddell, J.W., Hamon, M., 2008. Diagnostic accuracy of in-stent coronary restenosis detection with multislice spiral computed tomography: A meta-analysis. Eur. Radiol. 18, 217-225.

Hug, J., Nagel, E., Bornstedt, A., Schnackenburg, B., Oswald, H., Fleck, E., 2000. Coronary arterial stents: Safety and artifacts during MR imaging. Radiology. 216, 781-787. 
Kefer, J.M., Coche, E., Vanoverschelde, J.L., Gerber, B.L., 2007. Diagnostic accuracy of 16-slice multidetector-row CT for detection of in-stent restenosis vs detection of stenosis in nonstented coronary arteries. Eur. Radiol. 17, 87-96.

Kim, W.Y., Danias, P.G., Stuber, M., Flamm, S.D., Plein, S., Nagel, E., Langerak, S.E., Weber, O.M., Pedersen, E.M., Schmidt, M., Botnar, R.M., Manning, W.J., 2001. Coronary magnetic resonance angiography for the detection of coronary stenoses. N. Engl. J. Med. 345, $1863-1869$.

Kitagawa, T., Fujii, T., Tomohiro, Y., Maeda, K., Kobayashi, M., Kunita, E., Sekiguchi, Y., 2006. Noninvasive assessment of coronary stents in patients by 16-slice computed tomography. Int. J. Cardiol. 109, 188-194.

Krüger, S., Mahnken, A.H., Sinha, A.M., Borghans, A., Dedden, K., Hoffmann, R., Hanrath, P., 2003. Multislice spiral computed tomography for the detection of coronary stent restenosis and patency. Int. J. Cardiol. 89, 167-172.

Maintz, D, Juergens, K.U., Wichter, T., Grude, M., Heindel, W., Fischbach, R., 2003a. Imaging of coronary artery stents using multislice computed tomography: In vitro evaluation. Eur. Radiol.13, 830-835.

Maintz, D., Grude, M., Fallenberg, E.M., Heindel, W., Fischbach, R., 2003b. Assessment of coronary arterial stents by multislice-CT angiography. Acta Radiol. 44, 597-603.

Möhlenkamp, S., Pump, H., Baumgart, D., Haude, M., Grönemeyer, D.H., Seibel, R.M., Schwartz, R.S., Erbel, R., 1999. Minimally invasive evaluation of coronary stents with electron beam computed tomography: In vivo and in vitro experience. Catheter. Cardiovasc. Interv. 48 , $39-47$.

Pump, H., Möhlenkamp, S., Sehnert, C.A., Schimpf, S.S., Schmidt, A., Erbel, R., Grönemeyer, D.H., Seibel, R.M., 2000. Coronary arterial stent patency: Assessment with electron-beam CT. Radiology. 214, 447-452.

Scanlon, P.J., Faxon, D.P., Audet, A.M., Carabello, B., Dehmer, G.J., Eagle, K.A., Legako, R.D., Leon, D.F., Murray, J.A., Nissen, S.E., Pepine, C.J., Watson, R.M., Ritchie, J.L., Gibbons, R.J., Cheitlin, M.D., Gardner, T.J., Garson, A. Jr., Russell, R.O. Jr., Ryan, T.J., Smith, S.C. Jr., 1999. ACC/AHA guidelines for coronary angiography. A report of the American College of Cardiology/American Heart Association Task Force on practice guidelines (Committee on Coronary Angiography). Developed in collaboration with the Society for Cardiac Angiography and Interventions. J. Am. Coll. Cardiol. 33, 1756-824.

Schuijf, J.D, Bax, J.J., Jukema, J.W., Lamb, H.J., Warda, H.M., Vliegen, H.W., de Roos, A., van der, Wall. E,E., 2004. Feasibility of assessment of coronary stent patency using 16-slice computed tomography. Am. J. Cardiol. 94, 427-430.

Soon, K.H., Cox, N., Chaitowitz, I., Selvanayagam, J.B., Farouque, O., MacGregor, L., Bell, K.W., Lim, Y.L.,2007. Non-invasive computed tomography angiography in the assessment of coronary stent patency: An Australian experience. Intern. Med. J. 37, 360-364.

Sun, Z., Davidson, R., Lin, C.H., 2009. Multi-detector row CT angiography in the assessment of coronary in-stent restenosis: A systematic review. Eur. J. Radiol. 69, 489-495.

Sun Z, Choo GH, NG KH., 2012. Coronary CT angiography: Current status and continuing challenges. Br J Radiol. 85, 495-510.

Watanabe, M., Uemura, S., Iwama, H., Okayama, S., Takeda, Y., Kawata, H., Horii, M., Nakajima, T., Hirohashi, S., Kichikawa, K., Ookura, A., Saito, Y., 2006. Usefulness of 16-slice multislice spiral computed tomography for follow-up study of coronary stent implantation. Circ. J. 70, 691-697. 